\title{
Clinical usefulness of psoas muscle thickness for the diagnosis of sarcopenia in patients with liver cirrhosis
}

\author{
Dae Hoe Gu', Moon Young Kim², Yeon Seok Seo', Sang Gyune Kim³, Han Ah Lee', Tae Hyung Kim1', Young Kul Jung', \\ Altay Kandemir', Ji Hoon Kim', Hyunggin An ${ }^{5}$, Hyung Joon Yim', Jong Eun Yeon', Kwan Soo Byun', and Soon Ho Um \\ 'Department of Internal Medicine, Korea University College of Medicine, Seoul; ${ }^{2}$ Department of Internal Medicine, Yonsei University \\ Wonju College of Medicine, Wonju; ${ }^{3}$ Department of Internal Medicine, Soonchunhyang University Bucheon Hospital, Bucheon, Korea; \\ ${ }^{4}$ Department of Gastroenterology, Adnan Menderes University Medical School, Aydin, Turkey; ${ }^{5}$ Department of Biostatistics, Korea \\ University College of Medicine, Seoul, Korea
}

Background/Aims: The most widely used method for diagnosing sarcopenia is the skeletal muscle index (SMI). Several studies have suggested that psoas muscle thickness per height (PMTH) is also effective for detecting sarcopenia and predicting prognosis in patients with cirrhosis. The aim of this study was to evaluate the optimal cutoff values of PMTH for detecting sarcopenia in cirrhotic patients.

Methods: All cirrhotic patients who underwent abdominal computed tomography (CT) scan including L3 and umbilical levels for measuring SMI and transverse psoas muscle thickness, respectively, were included. Two definitions of sarcopenia were used: (1) sex-specific cutoffs of SMI $\left(\leq 52.4 \mathrm{~cm}^{2} / \mathrm{m}^{2}\right.$ in men and $\leq 38.5 \mathrm{~cm}^{2} / \mathrm{m}^{2}$ in women) for SMIsarcopenia and (2) cutoff of PMTH $(<16.8 \mathrm{~mm} / \mathrm{m})$ for PMTH-sarcopenia.

Results: Six hundred fifty-three patients were included. The average age was $53.6 \pm 10.2$ years, and 499 patients (76.4\%) were men. PMTH correlated well with SMI in both men and women $(P<0.001)$. Two hundred forty-one (36.9\%) patients met the criteria for SMI-sarcopenia. The best PMTH cutoff values for predicting SMI-sarcopenia were $17.3 \mathrm{~mm} / \mathrm{m}$ in men and $10.4 \mathrm{~mm} / \mathrm{m}$ in women, and these were defined as sex-specific cutoffs of PMTH (SsPMTH). The previously published cutoff of PMTH was defined as sex-nonspecific cutoff of PMTH (SnPMTH). Two hundred thirty (35.2\%) patients were diagnosed with SsPMTH-sarcopenia, and 280 (44.4\%) patients were diagnosed with SnPMTH-sarcopenia. On a multivariate Cox regression analysis, SsPMTH-sarcopenia (hazard ratio [HR], 1.944; 95\% confidence interval [Cl], 1.144-3.304; $P=0.014$ ) was significantly associated with mortality, while SnPMTH-sarcopenia was not $(\mathrm{HR}, 1.446 ; 95 \% \mathrm{Cl}, 0.861-2.431 ; P=0.164)$.

Conclusions: PMTH was well correlated with SMI in cirrhotic patients. SsPMTH-sarcopenia was an independent predictor of mortality in these patients and more accurately predicted mortality compared to SnPMTH-sarcopenia. (Clin Mol Hepatol 2018;24:319-330)

Keywords: Sarcopenia; Cirrhosis; Prognosis; Psoas muscle

\begin{abstract}
Abbreviations:
ALT, alanine aminotransferase; AST, aspartate aminotransferase; AUROC, area under the receiver operating characteristic curve; $\mathrm{BMI}$, body mass index; $\mathrm{Cl}$, confidence interval; $\mathrm{Cr}$, creatinine; $\mathrm{CT}$, computed tomography; INR, international normalized ratio; HR, hazard ratio; MELD, model for end-stage liver disease; $O R$, odds ratio; PMTH, psoas muscle thickness/height; SMI, skeletal muscle index SnPMTH, sex-nonspecific psoas muscle thickness/height; SSPMTH, sex-specific psoas muscle thickness/height

*Both authors contributed equally to this work as joint first authors.
\end{abstract}

Corresponding author: Yeon Seok Seo

Department of Internal Medicine, Korea University College of Medicine, 73 Inchon-ro, Seongbuk-gu, Seoul 02841, Korea

Tel: +82-2-920-6608, Fax: +82-2-953-1943, E-mail: drseo@korea.ac.kr http://orcid.org/0000-0003-4171-6331

\section{Sang Gyune Kim}

Department of Internal Medicine, Soonchunhyang University Bucheon Hospital, Soonchunhyang University College of Medicine, 170 Jomaru-ro, Bucheon 14584, Korea

Tel: +82-32-621-5079, Fax: +82-32-621-5080

E-mail:mcnulty@schmc.ac.kr

http://orcid.org/0000-0001-8694-777X 


\begin{abstract}
Study Highlights
Sarcopenia is a common complication in patients with liver cirrhosis. Although the smooth muscle index (SMI) is the most widely used technique for the diagnosis of sarcopenia, delineating the surface of muscles is relatively complex, and specific software is need for computation. Previous studies suggested that psoas muscle thickness represents muscle mass and psoas muscle thickness/height (PMTH) is associated with prognosis of patients with liver cirrhosis. In this study, PMTH was a good substitute for SMI and an independent prognostic factor for mortality in cirrhotic patients. In addition, applying different cutoff values based on sex improves its prognostic value.
\end{abstract}

\section{INTRODUCTION}

Sarcopenia is characterized by a decline in muscle mass, muscle strength, and physical activity.' In the past, sarcopenia was only considered part of the aging process. ${ }^{2}$ However, recent studies have shown that sarcopenia is associated with impaired physical capability, ${ }_{1}^{3-5}$ poor quality of life, adverse metabolic effects, falls, ${ }^{6}$ disability, mortality, ${ }^{7,8}$ and high healthcare expenditures. ${ }^{9}$ Therefore, sarcopenia is increasingly recognized as a progressive disease frequently associated with various comorbidities and mortality. ${ }^{10}$

Malnutrition is common in patients with advanced liver diseases, and malnutrition and sarcopenia frequently occur in patients with liver cirrhosis. ${ }^{11-15} \mathrm{~A}$ number of studies have reported that, in cases of cirrhosis, sarcopenia is an independent prognostic factor of prognosis. ${ }^{13-21}$ However, objective assessment of muscle mass in cirrhotic patients remains challenging. Various definitions and measurement methods have been used to quantify and diagnose sarcopenia. However, there is still no consensus on measurement and cutoff values. ${ }^{22}$

Radiological image analysis is being increasingly utilized to diagnose sarcopenia in patients with cirrhosis. ${ }^{10}$ The skeletal muscle index (SMI), assessed by the cross-sectional area of several muscles on computed tomography (CT) at the L3 vertebral level, is the most widely used technique. SMI has been shown to be an independent predictor of mortality in cirrhotic patients. ${ }^{23-26}$ Abdominal muscles, including the psoas muscle, are appropriate to use for the assessment of skeletal muscle mass, because abdominal muscle mass, unlike appendicular muscle mass, is relatively independent of activity level. ${ }^{10}$ However, delineating the surface of different groups of muscles is relatively complex, takes time and specific software is needed for computation. Therefore, SMI is hardly generalizable to outpatients.

A recent study used psoas muscle thickness measured on $\mathrm{CT}$ to diagnose sarcopenia. ${ }^{27}$ They measured transverse psoas muscle thickness, defined as diameter of the psoas muscle perpendicular to the longest axial diameter of the muscle at the umbilical level, and then normalized the value to the patient's height by dividing transverse psoas muscle thickness by height. This psoas muscle thickness/height (PMTH) was easily calculated as muscle mass and was determined to be associated with mortality in patients with cirrhosis. This method does not require specific software and can be calculated quickly. However, the study did not correlate PMTH with other measurements, such as psoas muscle area or SMI. Also, sex differences in muscle mass were not considered, and $16.8 \mathrm{~mm} / \mathrm{m}$ was suggested as a cutoff value for the definition of sarcopenia in all patients. Our study was performed to measure how well PMTH predicts outcomes in cirrhotic patients and to establish optimal PMTH cutoff values for detecting sarcopenia by sex.

\section{MATERIALS AND METHODS}

\section{Patients}

We included all patients with liver cirrhosis who underwent an abdominal CT and laboratory tests at the Korea University Anam Hospital, Soonchunhyang University Bucheon Hospital, and Wonju Severance Christian Hospital. Patients with hepatocellular carcinoma and parenchymal renal disease were excluded. Liver cirrhosis was diagnosed histologically or clinically. The protocol was approved by the Human Ethics Committee of the Korea University Anam Hospital and conformed to the ethical guidelines of the 2008 Declaration of Helsinki. A waiver of consent was obtained, and the patient records were anonymized and de-identified prior to analysis.

\section{Data collection}

Data on age, sex, height, body weight, body mass index (BMI), underlying liver diseases, and results of the baseline laboratory tests were collected for each patient. Baseline laboratory tests consisted of platelet count, international normalized ratio (INR), serum aspartate aminotransferase (AST), alanine aminotransferase (ALT), bilirubin, albumin, creatinine $(\mathrm{Cr})$, and sodium levels. The 
model for end-stage liver disease (MELD) score was calculated using the following equation: $9.57 \times \log _{\mathrm{e}}$ (creatinine, $\left.\mathrm{mg} / \mathrm{dL}\right)+3.78 \times$ $\log _{\mathrm{e}}$ (bilirubin, $\mathrm{mg} / \mathrm{dL}$ )+11.2x $\log _{\mathrm{e}}\left(\right.$ INR) $+6.43 .{ }^{28}$ Clinical, laboratory, and radiological data used for the analysis were obtained within 1 week of the $\mathrm{CT}$ image used to quantify skeletal muscle mass.

\section{Measurement of skeletal muscle area and definition of sarcopenia}

Two axial images one at the L3 vertebral body and one at the level of the umbilicus were selected for each case. To determine PMTH, axial and transverse thickness of right psoas muscle was measured at the level of the umbilicus. Axial psoas muscle thickness was defined as the largest diameter of psoas muscle on an axial view (Fig. 1). Transversal psoas muscle thickness was defined as the diameter of psoas muscle perpendicular to the axial diameter. Psoas muscle thickness was normalized to the patient's height. For SMI, skeletal muscle area $\left(\mathrm{cm}^{2}\right)$ was measured by a trained operator with the NIH ImageJ software which enables specific tissue demarcation using previously reported Hounsfield unit thresholds (for skeletal muscle, -29 to +150 ). ${ }^{29}$ The skeletal muscle area at L3 vertebra was normalized to the patient's height squared $\left(\mathrm{m}^{2}\right)$.

Sarcopenia was defined by previously published cutoffs: (1) sexspecific cutoffs of SMI $\left(\leq 52.4 \mathrm{~cm}^{2} / \mathrm{m}^{2}\right.$ in men and $\leq 38.5 \mathrm{~cm}^{2} / \mathrm{m}^{2}$ in women) for SMI-sarcopenia ${ }^{14}$; (2) cutoff of PMTH $(<16.8 \mathrm{~mm} / \mathrm{m})$ for PMTH-sarcopenia (sex-nonspecific PMTH sarcopenia, SnPMTHsarcopenia). ${ }^{27}$

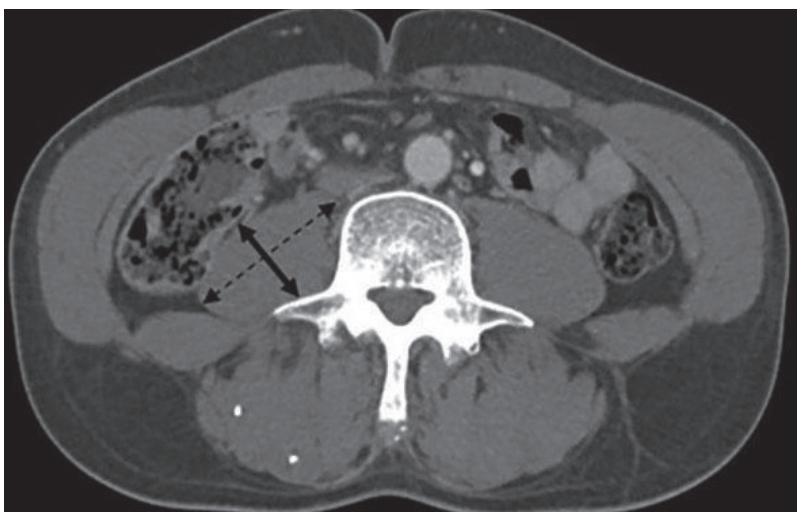

Figure 1. Measurement of psoas muscle thickness/height at the level of the umbilicus on a computed tomography (CT) scan image. Axial CT scan at the level of the umbilicus in a patient with hepatitis B virus-related cirrhosis and a model for end-stage liver disease (MELD) score of 10 with no ascites. Psoas muscle thickness corresponds to the diameter of transversal psoas muscle (left-right arrow) perpendicular to the axial diameter (dotted left-right arrow).

\section{Statistical analysis}

Statistical analyses were performed using the Statistical Package for the Social Science (SPSS) version 20.0 for Windows (IBM Corp., Armonk, NY, USA). All data are expressed as either mean \pm standard deviation for continuous variables or number of patients (percentage) for categorical variables. Groups were compared using the chisquare test for categorical variables and Student's $t$-test for quantitative variables. The area under the receiver operating characteristic curve (AUROC) and 95\% confidence interval (CI) were used to find the optimal values of sex-specific psoas muscle thickness/height (SsPMTH)-sarcopenia to predict SMI-sarcopenia. Survival time was calculated as the time interval from the date of the abdominal CT scan to death or to the end of the follow-up. Patients who had liver transplantation were censored at the time of liver transplantation. Survival curves were constructed using the Kaplan-Meier method, and differences were assessed by the logrank test. A Cox regression analysis was performed to find which variables were predictors of mortality. Hazard ratios (HRs) and $95 \%$ Cls were calculated. A two-tailed $P$-value of less than 0.05 was considered statistically significant.

\section{RESULTS}

\section{Baseline characteristics}

Table 1 presents baseline characteristics of all included patients. A total of 653 patients were included. The average age was $53.6 \pm 10.2$ years and 499 patients (76.4\%) were men. Alcoholic liver disease was the most common underlying liver disease (326 patients, 49.9\%), followed by chronic viral hepatitis (269 patients, $41.0 \%)$. Most of the cause of chronic viral hepatitis was chronic hepatitis B (244 of 269 patients, 90.7\%). The average MELD score was $11.4 \pm 4.8$. Ascites was absent in 332 patients $(50.8 \%)$, mild in 197 patients (30.2\%), and moderate in 124 patients (19.0\%).

\section{Sarcopenia defined by SMI}

Two hundred forty-one (36.9\%) patients met the criteria for SMIsarcopenia. In this group, men and alcoholic liver disease were more common than those that did not meet the threshold for SMIsarcopenia (Table 2). Serum bilirubin level and the MELD score were significantly higher in patients with SMI-sarcopenia, while $\mathrm{BMI}$ and serum sodium level were significantly lower compared to 
Table 1. Baseline characteristics of all included patients with liver cirrhosis

\begin{tabular}{lc}
\hline & All patients $(\mathbf{n}=653)$ \\
\hline Age $($ years $)$ & $53.6 \pm 10.2$ \\
Male $(\mathrm{n}, \%)$ & $499(76.4)$ \\
BMI $\left(\mathrm{kg} / \mathrm{m}^{2}\right)$ & $23.6 \pm 3.8$ \\
PMTH $(\mathrm{mm} / \mathrm{m})$ & $17.4 \pm 4.0$ \\
Underlying liver disease $(\mathrm{n}, \%)$ & \\
Viral & $268(41.0)$ \\
Alcohol & $326(49.9)$ \\
Others & $59(9.0)$ \\
\hline Diabetes $(\mathrm{n}, \%)$ & $181(27.7)$ \\
\hline Platelet count $\left(\times 10^{9} / \mathrm{L}\right)$ & $103.6 \pm 64.6$ \\
INR & $1.3 \pm 0.3$ \\
\hline AST $(I \mathrm{U} / \mathrm{L})$ & $79.8 \pm 159.8$ \\
ALT $(\mathrm{IU} / \mathrm{L})$ & $60.0 \pm 78.5$ \\
\hline Bilirubin $(\mathrm{mg} / \mathrm{dL})$ & $2.5 \pm 3.8$ \\
\hline Albumin $(\mathrm{g} / \mathrm{dL})$ & $3.4 \pm 0.7$ \\
\hline Creatinine $(\mathrm{mg} / \mathrm{dL})$ & $0.8 \pm 0.3$ \\
\hline Sodium $(\mathrm{mEq} / \mathrm{L})$ & $137.6 \pm 4.0$ \\
\hline MELD score & $11.4 \pm 4.8$ \\
\hline
\end{tabular}

Values are presented as mean \pm SD or $n(\%)$.

BMI, body mass index; PMTH, psoas muscle thickness/height; INR, international normalized ratio; AST, aspartate aminotransferase; ALT, alanine aminotransferase; MELD, model for end stage liver disease.

those without SMI-sarcopenia. PMTH was significantly lower in the patients with SMI-sarcopenia compared to those without SMI-sarcopenia $(15.8 \pm 3.6 \mathrm{~mm} / \mathrm{m}$ vs. $18.4 \pm 4.0 \mathrm{~mm} / \mathrm{m}, P<0.001)$. On a multivariate binary regression analysis, the presence of SMI-based sarcopenia was independently associated with male sex (odds ratio [OR], 7.657; 95\% Cl, 4.190-13.992; $P<0.001$ ), lower BMI (OR, $0.709 ; 95 \% \mathrm{Cl}, 0.661-0.761 ; P<0.001)$, and serum bilirubin level (OR, 1.068; 95\% Cl, 1.013-1.125; $P=0.015)$ (Table 3).

\section{Sarcopenia defined by PMTH}

PMTH was significantly higher in men than in women $(18.1 \pm 3.8$ $\mathrm{mm} / \mathrm{m}$ vs. $15.2 \pm 3.9 \mathrm{~mm} / \mathrm{m}, P<0.001)$. PMTH was well correlated with SMI (Pearson's correlation coefficient, 0.526; $P<0.001$ ) (Fig. 2A). The Pearson's correlation coefficient differed between men $(0.505, P<0.001)$ (Fig. 2B) and women $(0.380, P<0.001)$ (Fig. 2C). Following previous cutoff value, PMTH was less than $16.8 \mathrm{~mm} / \mathrm{m}$ in 290 patients (44.4\%) and they were defined as sex-nonspecific psoas muscle thickness/height (SnPMTH)-sarcopenia.

The AUROCs of PMTH for predicting SMI-sarcopenia were 0.779 $(95 \% \mathrm{Cl}, 0.739-0.819)$ in men (Fig. 3A) and $0.842(95 \% \mathrm{Cl}, 0.772-$ 0.912 ) in women (Fig. 3B). The best cutoff values for predicting SMI-sarcopenia were $17.3 \mathrm{~mm} / \mathrm{m}$ in men (sensitivity, $65.3 \%$; specificity, $76.3 \%$ ) and $10.4 \mathrm{~mm} / \mathrm{m}$ in women (sensitivity, $31.2 \%$; specificity, 90.6\%). Using these cutoff values, sarcopenia was diagnosed in 230 patients (35.2\%) and they were defined as SsPMTHsarcopenia.

When sarcopenia was diagnosed using the SnPMTH-sarcopenia, more women were defined as sarcopenic. Serum bilirubin level was significantly higher in patients with SnPMTH-sarcopenia, while $B M I$, serum albumin, and creatinine levels were significantly lower. The MELD score did not differ between the two groups (11.8 \pm 4.9 vs. $11.1 \pm 4.7, P=0.083$ ) (Table 2). On multivariate binary regression analysis, the presence of SnPMTH-sarcopenia was independently associated with female sex $(O R, 4.794 ; 95 \% \mathrm{Cl}$, 3.046-7.545; $P<0.001)$, lower BMI (OR, 0.777; $95 \% \mathrm{Cl}, 0.734-$ $0.822 ; P<0.001)$, and serum albumin level $(\mathrm{OR}, 0.486 ; 95 \% \mathrm{Cl}$ 0.366-0.645; $P<0.001$ ) (Table 4).

When sarcopenia was defined with SsPMTH-sarcopenia, men and those with alcoholic liver disease were more frequently defined as sarcopenic. Serum bilirubin level and the MELD score were significantly higher in patients with SsPMTH-sarcopenia, while BMI and serum sodium level were significantly lower (Table 2). On multivariate binary regression analysis, the presence of SsPMTH-sarcopenia was independently associated with sex (OR, 5.558; 95\% $\mathrm{Cl}, 3.109-9.937 ; P<0.001)$, lower BMI (OR, 0.759; $95 \% \mathrm{Cl}, 0.712$ $0.810 ; P<0.001)$, alcoholic liver disease $(\mathrm{OR}, 1.592 ; 95 \% \mathrm{Cl}, 1.080$ 2.346; $P=0.019)$, and serum albumin level $(O R, 0.417 ; 95 \% \mathrm{Cl}$, 0.288-0.603; $P<0.001$ ) (Table 5).

\section{Mortality}

Cumulative survival rate differed significantly according to the presence of sarcopenia (Fig. 4). With the definition of sarcopenia by SMI, survival rates at 1, 2, and 3 years were $97.7 \%, 93.3 \%$, and $88.3 \%$, respectively, in patients without sarcopenia, while those were $86.2 \%, 81.0 \%$, and $78.8 \%$, respectively, in patients with sarcopenia $(P<0.001)$ (Fig. $4 A)$. With the definition of sarcopenia by sex-nonspecific cutoff of PMTH the survival rates at 1, 2, and 3 years were $95.3 \%, 91.5 \%$, and $88.4 \%$, respectively, in patients without sarcopenia, while those were $91.4 \%, 85.6 \%$, and $80.6 \%$, respectively, in patients with sarcopenia ( $P=0.024)$ (Fig. 4B). With the definition of sarcopenia by sex-specific cutoff of PMTH, survival rates 
Dae Hoe Gu, et al.

Psoas muscle thickness for the diagnosis of sarcopenia

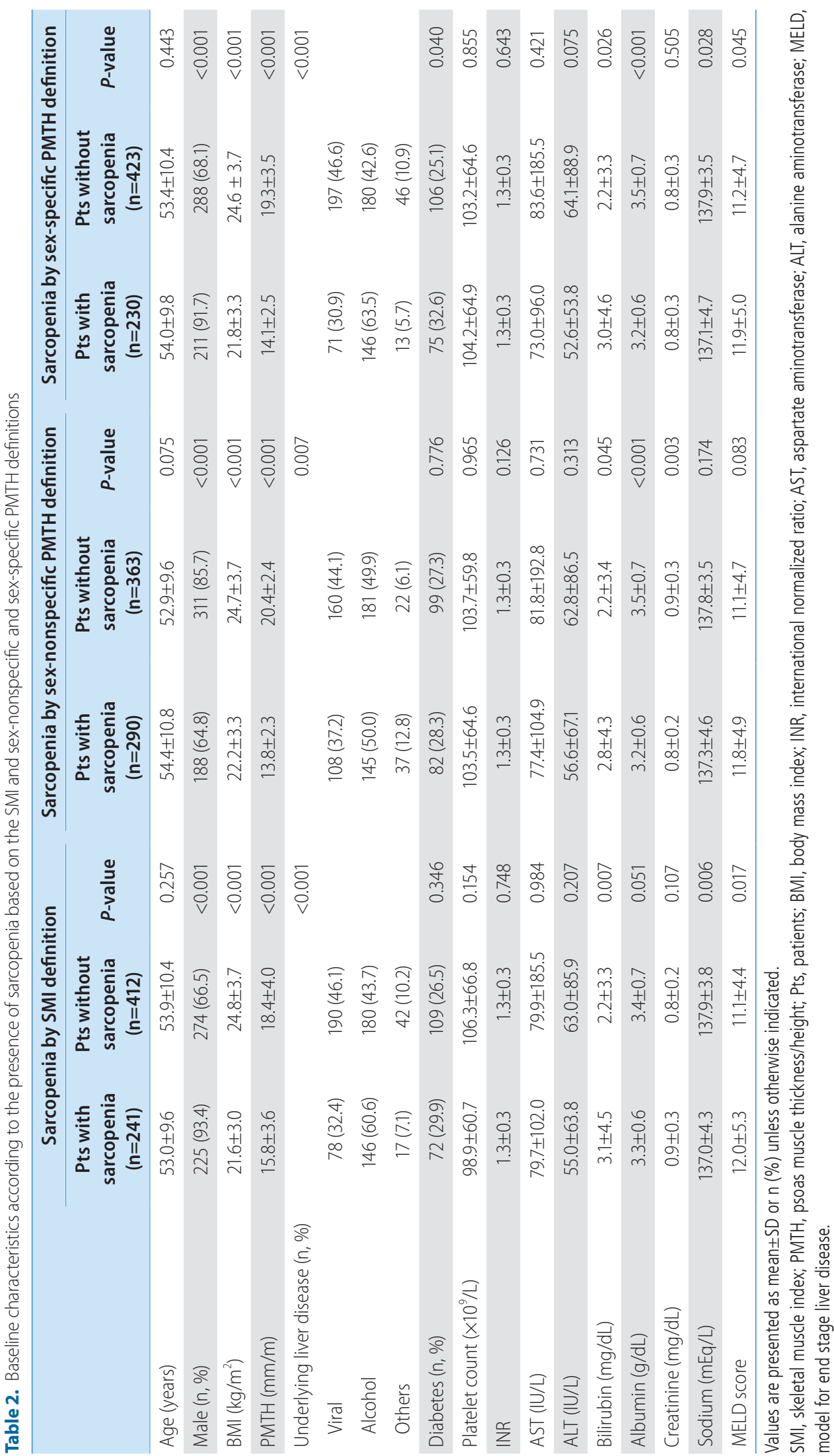


Table 3. Univariate and multivariate binary regression analyses for the presence of sarcopenia based on the SMI definition

\begin{tabular}{|c|c|c|c|c|c|}
\hline & \multirow{2}{*}{ Rating } & \multicolumn{2}{|c|}{ Univariate } & \multicolumn{2}{|c|}{ Multivariate } \\
\hline & & OR $(95 \% \mathrm{Cl})$ & $P$-value & OR $(95 \% \mathrm{Cl})$ & $P$-value \\
\hline Age & Years & $0.991(0.976-1.007)$ & 0.274 & & \\
\hline Sex & $0=$ female; $1=$ male & $7.083(4.099-12.237)$ & $<0.001$ & 7.657 (4.190-13.992) & $<0.001$ \\
\hline $\mathrm{BMI}$ & $\mathrm{kg} / \mathrm{m}^{2}$ & $0.734(0.690-0.782)$ & $<0.001$ & $0.709(0.661-0.761)$ & $<0.001$ \\
\hline Underlying liver disease & $0=$ other; $1=$ alcohol & $1.981(1.433-2.737)$ & $<0.001$ & 1.337 (0.907-1.1971) & 0.142 \\
\hline Diabetes & $0=$ no; $1=$ yes & $1.184(0.833-1.684)$ & 0.346 & & \\
\hline Platelet count & $\times 10^{9} / \mathrm{L}$ & $0.998(0.996-1.001)$ & 0.155 & & \\
\hline INR & Ratio & $1.083(0.665-1.764)$ & 0.748 & & \\
\hline AST & IU/L & 1.000 (0.999-1.001) & 0.984 & & \\
\hline ALT & $\mathrm{IU} / \mathrm{L}$ & $0.999(0.996-1.001)$ & 0.212 & & \\
\hline Bilirubin & $\mathrm{mg} / \mathrm{dL}$ & $1.063(1.018-1.110)$ & 0.006 & $1.068(1.013-1.125)$ & 0.015 \\
\hline Albumin & $\mathrm{g} / \mathrm{dL}$ & $0.784(0.613-1.002)$ & 0.052 & & \\
\hline Creatinine & $\mathrm{mg} / \mathrm{dL}$ & 1.677 (0.909-3.094) & 0.098 & & \\
\hline Sodium & $\mathrm{mEq} / \mathrm{L}$ & $0.943(0.906-0.982)$ & 0.005 & 1.337 (0.907-1.971) & 0.142 \\
\hline
\end{tabular}

SMI, skeletal muscle index; OR, odds ratio; $\mathrm{Cl}$, confidence interval; BMI, body mass index; INR, international normalized ratio; AST, aspartate aminotransferase; ALT, alanine aminotransferase.

at 1, 2, and 3 years were $96.2 \%, 92.4 \%$, and $89.7 \%$, respectively, in patients without sarcopenia, while those were $90.2 \%, 84.1 \%$, and $78.5 \%$, respectively, in patients with sarcopenia $(P=0.002)$ (Fig. 4C).

On a univariate Cox regression analysis, the presence of sarcopenia was significantly associated with mortality when any definition was applied. In addition, underlying liver disease, platelet count, INR, serum bilirubin, albumin, creatinine, and sodium levels were significantly associated with mortality (Table 6).

A multivariate Cox regression analysis was performed after adjusting for underlying liver disease, platelet count, INR, bilirubin, albumin, $\mathrm{Cr}$, and sodium levels. SMI-sarcopenia (HR, 1.841; 95\% $\mathrm{Cl}, 1.072-3.164 ; P=0.027)$ and SsPMTH-sarcopenia $(H R, 1.818$; $95 \% \mathrm{Cl}, 1.056-3.130 ; P=0.031)$ were significantly associated with mortality, but there was no significant association between SnPMTHsarcopenia and mortality (HR, 1.613; 95\% Cl, 0.950-2.739; $P=0.077)$ (Table 7).

\section{DISCUSSION}

In this study, PMTH was well correlated with SMI, and the AUROC of PMTH for predicting sarcopenia defined by SMI was relatively high in both men and women suggesting that PMTH is a more simple, objective, and readily available method to assess skel- etal muscle mass. Similarly to previous studies, SMI-based sarcopenia was predictive of mortality, independently of other possible confounders. Interestingly, we found that sex-specific PMTH-based sarcopenia was significantly associated with mortality, while sexnonspecific PMTH-based sarcopenia was not.

A number of studies showed men had significantly more skeletal muscle mass than in women, even after adjustment for body weight and height. ${ }^{30-33}$ Moreover, the association between sarcopenia and functional decline is stronger in men than in women. ${ }^{34,35}$ These differences may be a natural result of the different exposure of sex hormones and the greater amount of physical activity in men. In muscle quantification studies based on magnetic resonance imaging, the muscle mass of the psoas major showed significant sex differences while the rectus abdominis, latissimus dorsi, or thigh muscles did not. ${ }^{36,37}$ Reasons for these sex differences are still unknown, but a greater functional requirement of trunk musculature might be associated with greater development of the psoas muscle in men who perform more physical activities than women. ${ }^{38-40}$ Also, during growth, women experience a lower increase in waist circumference than men. This morphological difference in the abdominal region might be associated with the difference in psoas muscle mass between the sexes. ${ }^{41-44}$

Recently, CT with image analysis programs is being increasingly used to assess skeletal muscle mass. Abdominal CT would be difficult to justify for muscle mass measurements alone due to cost and 

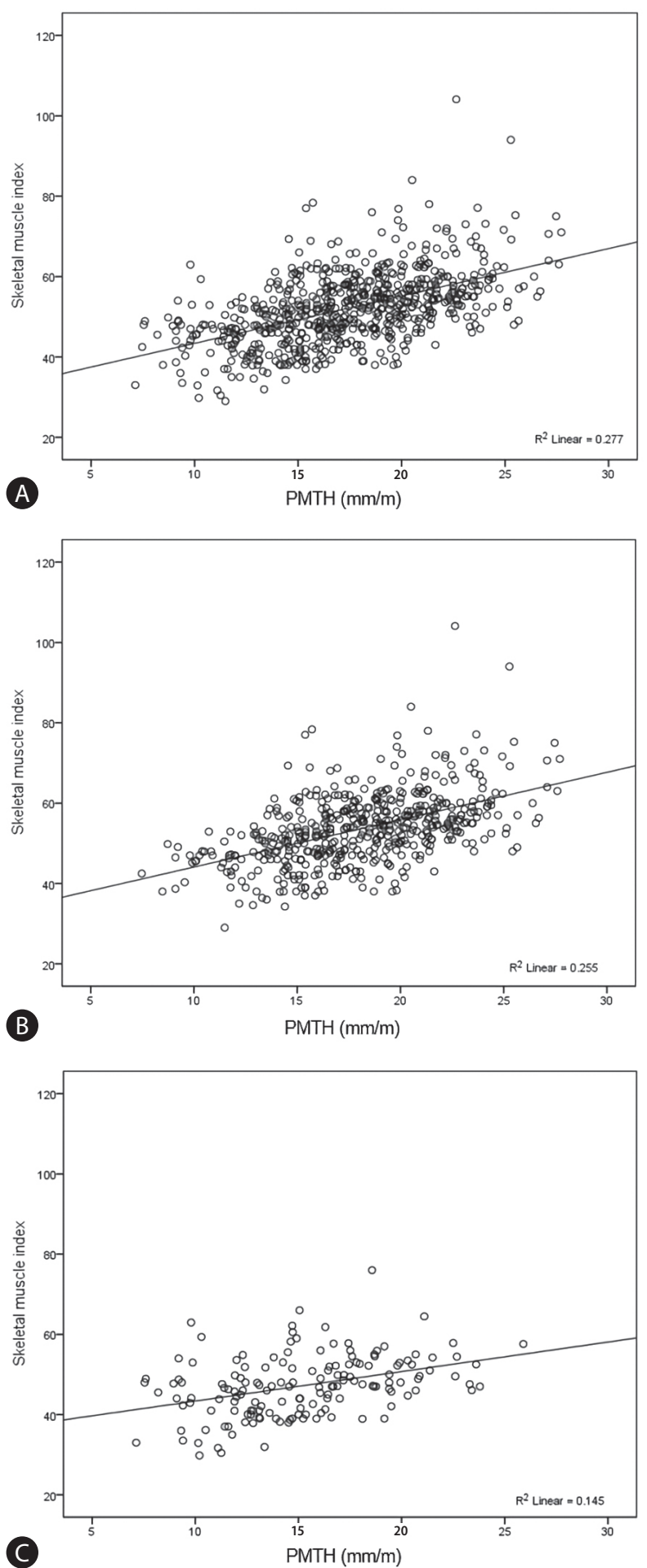

Figure 2. Correlation between psoas muscle thickness/height and skeletal muscle index. Correlation between psoas muscle thickness/height and skeletal muscle index in all enrolled patients (A), in men (B), and in women (C). PMTH was well correlated with SMI (Pearson's correlation coefficient, 0.526; $P<0.001)(A)$. The Pearson's correlation coefficient differed between men $(0.505, P<0.001)(B)$ and women $(0.380, P<0.001)(C)$. $\mathrm{PMTH}$, psoas muscle thickness/height; SMl, skeletal muscle index. radiation exposure. However, as most patients with liver cirrhosis have surveillance scans for detecting focal liver lesions, ${ }^{10}$ additional CT scans for measuring muscle mass are not usually needed.

Previous researchers have suggested that PMTH, measured on $\mathrm{CT}$ at the level of the umbilicus, may be predictive of mortality in cirrhotic patients independently of the MELD and MELD-Na scores. They also suggested that the optimal cutoff point of $16.8 \mathrm{~mm} / \mathrm{m}$ be used to predict mortality in both sexes. ${ }^{27}$ However, contrary to other studies, when this cutoff value was applied, sarcopenia was more frequent in women than in men. Besides, the mean MELD score (11.8 \pm 4.9 vs. $11.1 \pm 4.7, P=0.083)$ and mortality (HR, 1.613; $95 \% \mathrm{Cl}, 0.950-2.739 ; P=0.077)$ were not significantly different between patients with and without SnPMTH sarcopenia. These results could be indicative of weakness of SnPMTH in predicting prognosis of cirrhotic patients. As amount of muscle mass differs with gender (lower in females) ${ }^{25}$ after adjustment for height, applying different cutoff values based on sex would be more appropriate. Therefore, we found the sex-specific cutoffs of PMTH $(<17.3 \mathrm{~mm} / \mathrm{m}$ in men and $<10.4 \mathrm{~mm} / \mathrm{m}$ in women) to be the optimal values for predicting sarcopenia defined by SMI. Among 290 patients (44.4\%) classified as sarcopenic by SnPMTH, 60 (9.2\%) were classified as non-sarcopenic by SSPMTH. Sarcopenia defined by sex-specific PMTH (HR, $1.818 ; 95 \% \mathrm{Cl}, 1.056-3.130 ; P=0.031$ ) was significantly associated with mortality.

It is hard to compare the results of the previous study and our current study due to the disparities between the cohorts. First, mean MELD score was higher in the previous study $(16.0 \pm 7)$ than in our study (11.4 \pm 4.8$)$. This was because only patients registered for deceased donor liver transplantation were enrolled in the previous study, while we included all patients with liver cirrhosis who had an abdominal CT scan. Therefore, mortality at 6 months was higher in the previous study (12.3\%) than in our study (3.7\%). Second, the previous study included HCC patients (46\%), who were excluded in our study in consideration of the impact of HCC on mortality. Our data more clearly shows the clinical effect of sarcopenia in cirrhotic patients. Lastly, mean BMI $\left(23.6 \pm 3.8 \mathrm{~kg} / \mathrm{m}^{2}\right.$ vs. $\left.26.2 \pm 4.7 \mathrm{~kg} / \mathrm{m}^{2}\right)$ and mean PMTH was lower in our study. This disparity may have been caused by the differences in body size, lifestyle, and cultural background between Asian and Caucasian populations. ${ }^{45}$ However, the prevalence of sarcopenia is usually not higher in Asians compared to Caucasians, although mean BMI and mean muscle mass are lower in Asians. This difference may be due to the characteristics of older Asian people, who have walked and performed more physical activities since their early adulthood because of the underdeveloped living conditions and transportation. 


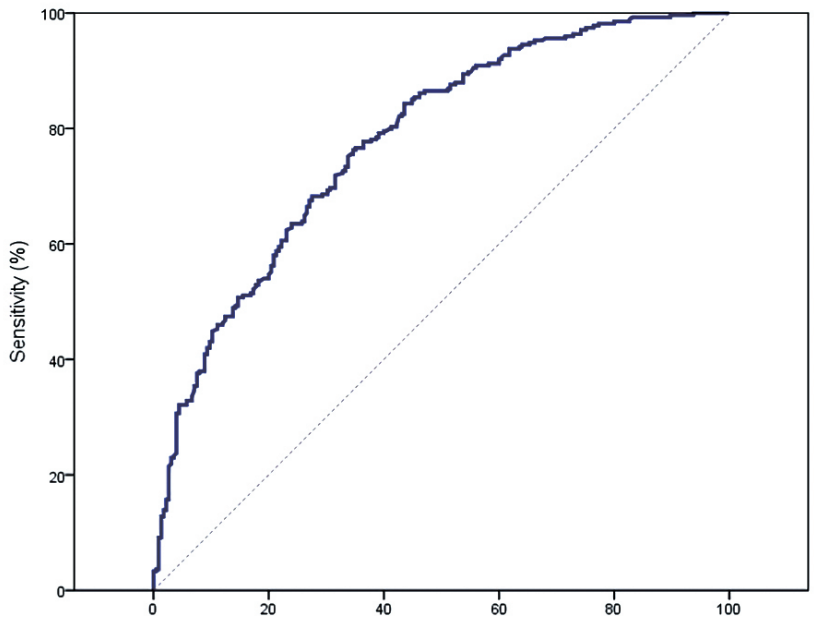

A 1 - Specificity (\%)

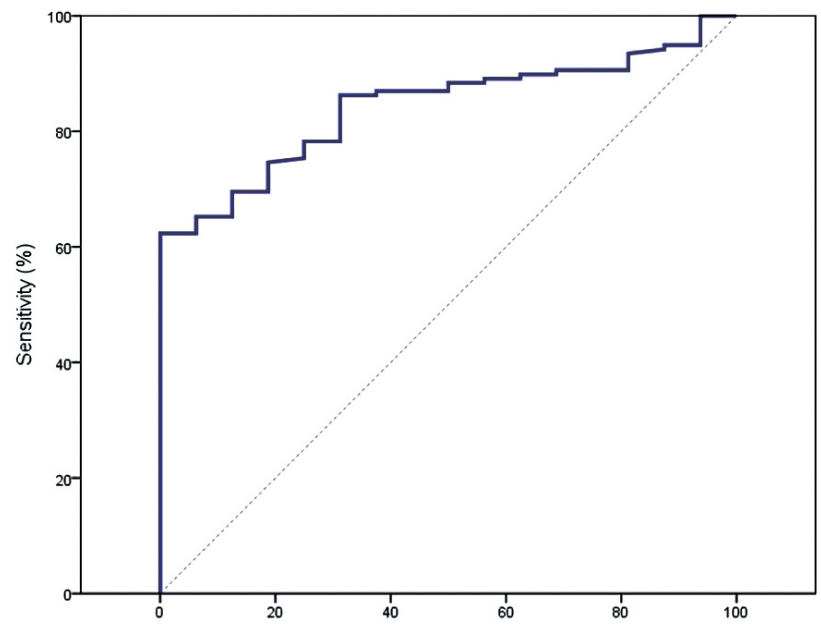

B

Figure 3. Area under the receiver operating characteristic curve for detecting sarcopenia. Area under the receiver operating characteristic curve for detecting sarcopenia defined by smooth muscle index based on psoas muscle thickness/height in men (A) and in women (B). The area under the receiver operating characteristic curve of psoas muscle thickness/height for predicting skeletal muscle index-sarcopenia was 0.779 ( $95 \%$ confidence interval [Cl], 0.739-0.819) in men (A) and 0.842 (95\% Cl, 0.772-0.912) in women (B).

Table 4. Univariate and multivariate binary regression analyses for the presence of sarcopenia based on the sex-nonspecific PMTH definition

\begin{tabular}{|c|c|c|c|c|c|}
\hline & \multirow{2}{*}{ Rating } & \multicolumn{2}{|c|}{ Univariate } & \multicolumn{2}{|c|}{ Multivariate } \\
\hline & & OR $(95 \% \mathrm{Cl})$ & $P$-value & OR $(95 \% \mathrm{Cl})$ & $P$-value \\
\hline Age & Years & 1.014 (0.999-1.030) & 0.072 & & \\
\hline Sex & $0=$ male; $1=$ female & 3.245 (2.219-4.744) & $<0.001$ & $4.794(3.046-7.545)$ & $<0.001$ \\
\hline $\mathrm{BMI}$ & $\mathrm{kg} / \mathrm{m}^{2}$ & $0.811(0.770-0.854)$ & $<0.001$ & $0.777(0.734-0.822)$ & $<0.001$ \\
\hline Underlying liver disease & $0=$ other $; 1=$ alcohol & 1.006 (0.738-1.369) & 0.972 & & \\
\hline Diabetes & $0=$ no; $1=$ yes & 1.051 (0.745-1.484) & 0.776 & & \\
\hline Platelet count & $\times 10^{9} / \mathrm{L}$ & $1.000(0.998-1.002)$ & 0.965 & & \\
\hline INR & Ratio & 1.451 (0.897-2.347) & 0.129 & & \\
\hline AST & $\mathrm{IU} / \mathrm{L}$ & 1.000 (0.999-1.001) & 0.733 & & \\
\hline ALT & $\mathrm{IU} / \mathrm{L}$ & 0.999 (0.997-1.001) & 0.316 & & \\
\hline Bilirubin & $\mathrm{mg} / \mathrm{dL}$ & $1.043(1.000-1.088)$ & 0.051 & & \\
\hline Albumin & $\mathrm{g} / \mathrm{dL}$ & $0.569(0.445-0.729)$ & $<0.001$ & $0.486(0.366-0.645)$ & $<0.001$ \\
\hline Creatinine & $\mathrm{mg} / \mathrm{dL}$ & $0.378(0.194-0.733)$ & 0.004 & $0.714(0.346-1.474)$ & 0.362 \\
\hline Sodium & $\mathrm{mEq} / \mathrm{L}$ & $0.973(0.935-1.011)$ & 0.161 & & \\
\hline
\end{tabular}

PMTH, psoas muscle thickness/height; OR, odds ratio; $\mathrm{Cl}$, confidence interval; BMI, body mass index; INR, international normalized ratio; AST, aspartate aminotransferase; ALT, alanine aminotransferase.

This study has several limitations. First, it is a retrospective study, and PMTH was measured by only one clinician. We could not evaluate inter-observer variance, although several studies have already shown relatively good agreement between observers. Second, PMTH has inherent limitations. Transverse psoas muscle thickness was measured at the level of the umbilicus, but the level of the umbilicus may vary if ascites is present. However, umbilical level can be easily identified on a CT scan than given level of the lumbar section, because sacralization of the $L 5$ vertebrae and lumbar wedge fractures in patients with osteoporosis make detection of given lumbar level difficult. ${ }^{27}$ In a recent systematic review and meta-analysis on the impact of CT-assessed skeletal muscle mass on outcomes in liver transplant candidates, ${ }^{22}$ only one study measured skeletal muscle mass using PMTH. ${ }^{27}$ Further studies validating the 
Table 5. Univariate and multivariate binary regression analyses for the presence of sarcopenia based on the sex-specific PMTH definition

\begin{tabular}{|c|c|c|c|c|c|}
\hline & \multirow{2}{*}{ Rating } & \multicolumn{2}{|c|}{ Univariate } & \multicolumn{2}{|c|}{ Multivariate } \\
\hline & & OR $(95 \% \mathrm{CI})$ & $P$-value & OR (95\% CI) & $P$-value \\
\hline Age & Years & $1.006(0.990-1.022)$ & 0.451 & & \\
\hline Sex & $0=$ female; $1=$ male & $5.206(3.120-8.687)$ & $<0.001$ & 5.558 (3.109-9.937) & $<0.001$ \\
\hline BMI & $\mathrm{kg} / \mathrm{m}^{2}$ & $0.780(0.737-0.827)$ & $<0.001$ & $0.759(0.712-0.810)$ & $<0.001$ \\
\hline Underlying liver disease & $0=$ other; $1=$ alcohol & $2.346(1.686-3.265)$ & $<0.001$ & $1.592(1.080-2.346)$ & 0.019 \\
\hline Diabetes & $0=$ no; $1=$ yes & 1.447 (1.017-2.059) & 0.040 & $1.513(0.997-2.297)$ & 0.052 \\
\hline Platelet count & $\times 10^{9} / \mathrm{L}$ & $1.000(0.998-1.003)$ & 0.855 & & \\
\hline INR & Ratio & $1.123(0.688-1.834)$ & 0.643 & & \\
\hline AST & IU/L & 0.999 (0.998-1.001) & 0.438 & & \\
\hline ALT & IU/L & $0.998(0.995-1.000)$ & 0.081 & & \\
\hline Bilirubin & $\mathrm{mg} / \mathrm{dL}$ & 1.052 (1.009-1.097) & 0.018 & $1.072(0.990-1.161)$ & 0.085 \\
\hline Albumin & $\mathrm{g} / \mathrm{dL}$ & $0.560(0.433-0.725)$ & $<0.001$ & $0.417(0.288-0.603)$ & $<0.001$ \\
\hline Creatinine & $\mathrm{mg} / \mathrm{dL}$ & $0.805(0.426-1.522)$ & 0.505 & & \\
\hline Sodium & $\mathrm{mEq} / \mathrm{L}$ & 0.952 (0.914-0.992) & 0.018 & $1.002(0.954-1.054)$ & 0.922 \\
\hline
\end{tabular}

PMTH, psoas muscle thickness/height; OR, odds ratio; $\mathrm{Cl}$, confidence interval; $\mathrm{BMI}$, body mass index; INR, international normalized ratio; AST, aspartate aminotransferase; ALT, alanine aminotransferase.
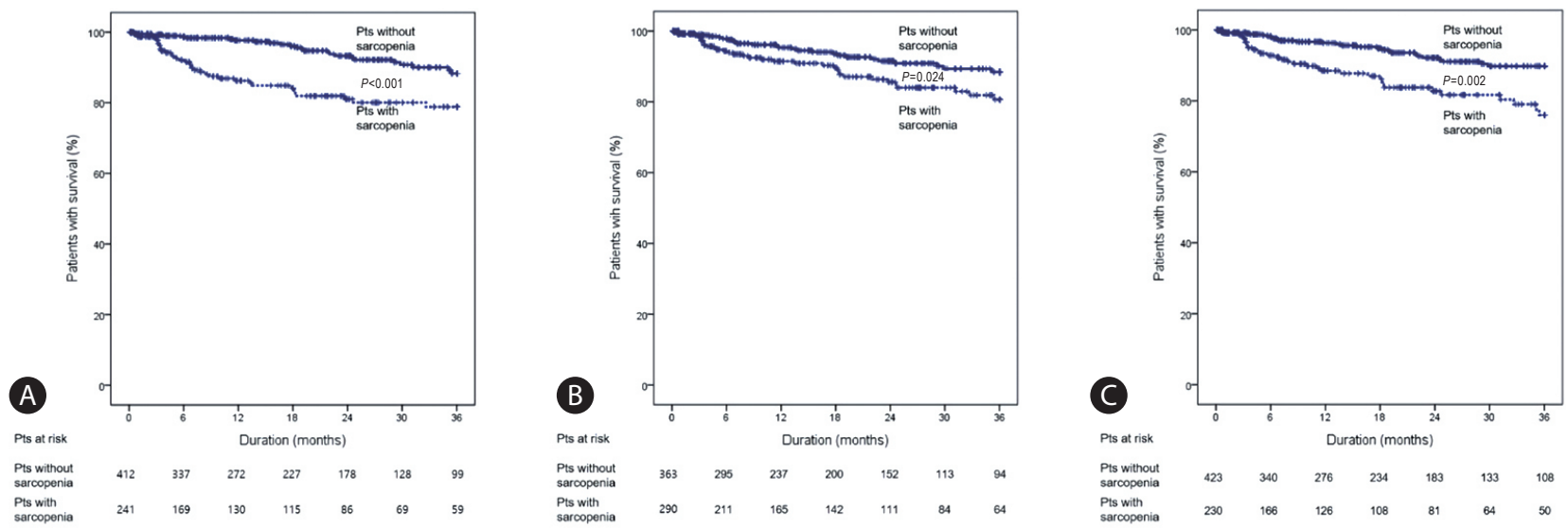

Figure 4. Kaplan-Meier analysis for survival according to the presence of sarcopenia. Kaplan-Meier curve for cumulative survival of patients with liver cirrhosis according to the presence of sarcopenia defined by smooth muscle index (A), sex-nonspecific PMTH (B), and sex-specific PMTH (C). With the definition of sarcopenia based on SMI, survival rates at 1, 2, and 3 years were 97.7\%, 93.3\%, and 88.3\%, respectively, in patients without sarcopenia, while they were $86.2 \%, 81.0 \%$, and $78.8 \%$ in patients with sarcopenia $(P<0.001)$ (A). With the definition of sarcopenia based on the sex-nonspecific cutoff of PMTH, the survival rates at 1, 2, and 3 years were $95.3 \%, 91.5 \%$, and $88.4 \%$, respectively, in patients without sarcopenia, while they were $91.4 \%$, $85.6 \%$, and $80.6 \%$ in patients with sarcopenia $(P=0.024)$ (B). With the definition of sarcopenia by sex-specific cutoff of PMTH, survival rates at 1,2 , and 3 years were $96.2 \%, 92.4 \%$, and $89.7 \%$, respectively, in patients without sarcopenia, while they were $90.2 \%, 84.1 \%$, and $78.5 \%$ in patients with sarcopenia ( $P=0.002)$ (C). Pts, patients; PMTH, psoas muscle thickness/height; SMl, skeletal muscle index.

efficacy of PMTH in patients with liver cirrhosis are needed. Lastly, assessment of muscle mass by $\mathrm{CT}$ scan also should be taken into account. Since muscle strength and amount of fat content is related to $\mathrm{CT}$ attenuation, $\mathrm{CT}$ attenuation is often lower in the muscles of cirrhotic patients compared to controls. ${ }^{25}$ It is uncertain whether sarcopenia in cirrhotic patients can be diagnosed with hounsfield unit alone. To overcome these limitations, numerous measurement methods have been suggested, however, few studies have directly compared the different methods so far.

In conclusion, we found that PMTH measured on a single axial 
Table 6. Univariate Cox regression analysis for mortality

\begin{tabular}{|c|c|c|c|}
\hline & Rating & HR $(95 \% \mathrm{Cl})$ & $P$-value \\
\hline SMI-sarcopenia & 0, no; 1 , yes & $2.710(1.622-4.528)$ & $<0.001$ \\
\hline SnPMTH-sarcopenia & 0, no; 1 , yes & 1.784 (1.071-2.973) & 0.026 \\
\hline SsPMTH-sarcopenia & 0, no; 1 , yes & $2.202(1.314-3.692)$ & 0.003 \\
\hline Age & Years & $1.009(0.984-1.034)$ & 0.495 \\
\hline Sex & 0, female; 1 , male & $1.873(0.922-3.805)$ & 0.083 \\
\hline $\mathrm{BMI}\left(\mathrm{kg} / \mathrm{m}^{2}\right)$ & $\mathrm{kg} / \mathrm{m}^{2}$ & $0.978(0.913-1.047)$ & 0.514 \\
\hline Underlying liver disease & 0 , other; 1 , alcohol & $1.629(0.972-2.730)$ & 0.064 \\
\hline Diabetes & 0, no; 1 , yes & $0.991(0.570-1.722)$ & 0.974 \\
\hline Platelet count & $\times 10^{9} / \mathrm{L}$ & $0.995(0.990-1.000)$ & 0.037 \\
\hline INR & Ratio & $3.798(2.114-6.825)$ & $<0.001$ \\
\hline AST & IU/L & $0.998(0.995-1.002)$ & 0.348 \\
\hline ALT & $I U / L$ & $0.999(0.996-1.002)$ & 0.520 \\
\hline Bilirubin & $\mathrm{mg} / \mathrm{dL}$ & $1.103(1.065-1.142)$ & $<0.001$ \\
\hline Albumin & $\mathrm{g} / \mathrm{dL}$ & $0.382(0.252-0.577)$ & $<0.001$ \\
\hline Creatinine & $\mathrm{mg} / \mathrm{dL}$ & 5.697 (2.552-12.721) & $<0.001$ \\
\hline Sodium & $\mathrm{mEq} / \mathrm{L}$ & $0.876(0.833-0.921)$ & $<0.001$ \\
\hline
\end{tabular}

HR, hazard ratio; $\mathrm{Cl}$, confidence interval; SMI, skeletal muscle index; SnPMTH, sex-nonspecific psoas muscle thickness/height; SsPMTH, sex-specific psoas muscle thickness/height; BMI, body mass index; INR, international normalized ratio; AST, aspartate aminotransferase; ALT, alanine aminotransferase.

Table 7. Cox regression analysis adjusted for underlying liver disease, platelet count, INR, and serum bilirubin, albumin, creatinine, and sodium levels

\begin{tabular}{lccc}
\hline & Rating & HR $(\mathbf{9 5} \% \mathbf{C l})$ & $\boldsymbol{P}$-value \\
\hline SMI-sarcopenia & 0, no; 1 , yes & $1.841(1.072-3.164)$ & 0.027 \\
SnPMTH-sarcopenia & 0, no; 1 , yes & $1.613(0.950-2.739)$ & 0.077 \\
SsPMTH-sarcopenia & 0, no; 1 , yes & $1.818(1.056-3.130)$ & 0.031 \\
\hline
\end{tabular}

INR, international normalized ratio; $\mathrm{HR}$, hazard ratio; $\mathrm{Cl}$, confidence interval; SMI, skeletal muscle index; SnPMTH, sex-nonspecific psoas muscle thickness/ height; SsPMTH, sex-specific psoas muscle thickness/height.

CT scan image at the level of the umbilicus may be a good substitute for SMI and an independent prognostic factor for mortality in cirrhotic patients. Additionally, applying different cutoff values based on sex improves the prognostic value of PMTH.

\section{Author contributions}

Study design: Yeon Seok Seo, Sang Gyune Kim

Acquisition and interpretation of data: Han Ah Lee, Tae Hyung Kim, Young Kul Jung, Moon Young Kim, Ji Hoon Kim

Drafting of the manuscript: Dae Hoe Gu, Moon Young Kim, Yeon Seok Seo
Critical revision of the manuscript for important intellectual content: Soon Ho Um, Altay Kandemir, Hyung Joon Yim, Jong Eun Yeon, Kwan Soo Byun

Statistical analysis: Hyunggin An, Yeon Seok Seo

\section{Conflicts of Interest}

The authors have no conflicts of interest to disclose.

\section{REFERENCES}

1. Delmonico MJ, Harris TB, Visser M, Park SW, Conroy MB, VelasquezMieyer $\mathrm{P}$, et al. Longitudinal study of muscle strength, quality, and adipose tissue infiltration. Am J Clin Nutr 2009;90:1579-1585.

2. Cruz-Jentoft AJ, Landi F, Topinková E, Michel JP. Understanding sarcopenia as a geriatric syndrome. Curr Opin Clin Nutr Metab Care 2010;13:1-7.

3. Auyeung TW, Lee JS, Leung J, Kwok T, Woo J. The selection of a screening test for frailty identification in community-dwelling older adults. J Nutr Health Aging 2014;18:199-203.

4. Tanimoto Y, Watanabe M, Sun W, Sugiura Y, Tsuda Y, Kimura M, et al. Association between sarcopenia and higher-level functional capacity in daily living in community-dwelling elderly subjects in Japan. 
Arch Gerontol Geriatr 2012;55:e9-e13.

5. Woo J, Leung J, Sham A, Kwok T. Defining sarcopenia in terms of risk of physical limitations: a 5-year follow-up study of 3,153 Chinese men and women. J Am Geriatr Soc 2009;57:2224-2231.

6. Karakelides H, Nair KS. Sarcopenia of aging and its metabolic impact. Curr Top Dev Biol 2005;68:123-148.

7. Cesari M, Pahor M, Lauretani F, Zamboni V, Bandinelli S, Bernabei R, et al. Skeletal muscle and mortality results from the InCHIANTI Study. J Gerontol A Biol Sci Med Sci 2009;64:377-384.

8. Newman AB, Kupelian V, Visser M, Simonsick EM, Goodpaster BH, Kritchevsky SB, et al. Strength, but not muscle mass, is associated with mortality in the health, aging and body composition study cohort. J Gerontol A Biol Sci Med Sci 2006;61:72-77.

9. Janssen I, Shepard DS, Katzmarzyk PT, Roubenoff R. The healthcare costs of sarcopenia in the United States. J Am Geriatr Soc 2004;52:80-85.

10. Dasarathy S, Merli M. Sarcopenia from mechanism to diagnosis and treatment in liver disease. J Hepatol 2016;65:1232-1244.

11. Alberino F, Gatta A, Amodio P, Merkel C, Di Pascoli L, Boffo G, et al. Nutrition and survival in patients with liver cirrhosis. Nutrition 2001;17:445450.

12. Campillo B, Paillaud E, Uzan I, Merlier I, Abdellaoui M, Perennec J, et al. Value of body mass index in the detection of severe malnutrition: influence of the pathology and changes in anthropometric parameters. Clin Nutr 2004;23:551-559.

13. Merli M, Giusto M, Gentili F, Novelli G, Ferretti G, Riggio O, et al. Nutritional status: its influence on the outcome of patients undergoing liver transplantation. Liver Int 2010;30:208-214.

14. Montano-Loza AJ, Meza-Junco J, Prado CM, Lieffers JR, Baracos VE, Bain VG, et al. Muscle wasting is associated with mortality in patients with cirrhosis. Clin Gastroenterol Hepatol 2012;10:166-173, 173.e1.

15. Tandon P, Ney M, Irwin I, Ma MM, Gramlich L, Bain VG, et al. Severe muscle depletion in patients on the liver transplant wait list: its prevalence and independent prognostic value. Liver Transpl 2012;18:12091216.

16. DiMartini A, Cruz RJ Jr, Dew MA, Myaskovsky L, Goodpaster B, Fox $K$, et al. Muscle mass predicts outcomes following liver transplantation. Liver Transpl 2013;19:1172-1180.

17. Hamaguchi Y, Kaido T, Okumura S, Kobayashi A, Fujimoto Y, Ogawa $K$, et al. Muscle steatosis is an independent predictor of postoperative complications in patients with hepatocellular carcinoma. World J Surg 2016;40:1959-1968.

18. Hanai T, Shiraki M, Ohnishi S, Miyazaki T, Ideta T, Kochi T, et al. Rapid skeletal muscle wasting predicts worse survival in patients with liver cirrhosis. Hepatol Res 2016;46:743-751.

19. Kim TY, Kim MY, Sohn JH, Kim SM, Ryu JA, Lim S, et al. Sarcopenia as a useful predictor for long-term mortality in cirrhotic patients with ascites. J Korean Med Sci 2014;29:1253-1259.

20. Masuda T, Shirabe K, Ikegami T, Harimoto N, Yoshizumi T, Soejima Y, et al. Sarcopenia is a prognostic factor in living donor liver transplantation. Liver Transpl 2014;20:401-407.

21. Wang CW, Feng S, Covinsky KE, Hayssen H, Zhou LQ, Yeh BM, et al. A comparison of muscle function, mass, and quality in liver transplant candidates: results from the functional assessment in liver transplantation study. Transplantation 2016;100:1692-1698.

22. van Vugt JL, Levolger S, de Bruin RW, van Rosmalen J, Metselaar HJ, IJzermans JN. Systematic review and meta-analysis of the impact of computed tomography-assessed skeletal muscle mass on outcome in patients awaiting or undergoing liver transplantation. Am J Transplant 2016;16:2277-2292.

23. Giusto M, Lattanzi B, Albanese C, Galtieri A, Farcomeni A, Giannelli V, et al. Sarcopenia in liver cirrhosis: the role of computed tomography scan for the assessment of muscle mass compared with dual-energy X-ray absorptiometry and anthropometry. Eur J Gastroenterol Hepatol 2015;27:328-334.

24. Glass C, Hipskind P, Tsien C, Malin SK, Kasumov T, Shah SN, et al. Sarcopenia and a physiologically low respiratory quotient in patients with cirrhosis: a prospective controlled study. J Appl Physiol (1985) 2013;114:559-565.

25. Tsien C, Garber A, Narayanan A, Shah SN, Barnes D, Eghtesad B, et al. Post-liver transplantation sarcopenia in cirrhosis: a prospective evaluation. J Gastroenterol Hepatol 2014;29:1250-1257.

26. Tsien C, Shah SN, McCullough AJ, Dasarathy S. Reversal of sarcopenia predicts survival after a transjugular intrahepatic portosystemic stent. Eur J Gastroenterol Hepatol 2013;25:85-93.

27. Durand F, Buyse S, Francoz C, Laouénan C, Bruno 0, Belghiti J, et al. Prognostic value of muscle atrophy in cirrhosis using psoas muscle thickness on computed tomography. J Hepatol 2014;60:1151-1157.

28. Wiesner R, Edwards E, Freeman R, Harper A, Kim R, Kamath P, et al. Model for end-stage liver disease (MELD) and allocation of donor livers. Gastroenterology 2003;124:91-96.

29. Mitsiopoulos N, Baumgartner RN, Heymsfield SB, Lyons W, Gallagher $D$, Ross R. Cadaver validation of skeletal muscle measurement by magnetic resonance imaging and computerized tomography. J Appl Physiol (1985) 1998;85:115-122.

30. Baumgartner RN, Waters DL, Gallagher D, Morley JE, Garry PJ. Predictors of skeletal muscle mass in elderly men and women. Mech Ageing Dev 1999;107:123-136.

31. Beneke R, Neuerburg J, Bohndorf K. Muscle cross-section measurement by magnetic resonance imaging. Eur J Appl Physiol Occup Physiol 1991;63:424-429.

32. Bevier WC, Wiswell RA, Pyka G, Kozak KC, Newhall KM, Marcus R. Relationship of body composition, muscle strength, and aerobic capacity to bone mineral density in older men and women. J Bone Miner Res 1989:4:421-432.

33. Janssen I, Heymsfield SB, Wang ZM, Ross R. Skeletal muscle mass and distribution in 468 men and women aged 18-88 yr. J Appl Physiol 
(1985) 2000;89:81-88.

34. Auyeung TW, Lee JS, Leung J, Kwok T, Woo J. Adiposity to muscle ratio predicts incident physical limitation in a cohort of 3,153 older adults--an alternative measurement of sarcopenia and sarcopenic obesity. Age (Dordr) 2013;35:1377-1385.

35. Dey DK, Bosaeus I, Lissner L, Steen B. Changes in body composition and its relation to muscle strength in 75-year-old men and women: a 5-year prospective follow-up study of the NORA cohort in Göteborg, Sweden. Nutrition 2009;25:613-619.

36. Hoshikawa Y, Muramatsu M, lida T, Ii N, Nakajima Y, Kanehisa H. Sex differences in the cross-sectional areas of psoas major and thigh muscles in high school track and field athletes and nonathletes. J Physiol Anthropol 2011;30:47-53.

37. Marras WS, Jorgensen MJ, Granata KP, Wiand B. Female and male trunk geometry: size and prediction of the spine loading trunk muscles derived from MRI. Clin Biomech (Bristol, Avon) 2001;16:38-46.

38. Bishop CA, Wine JJ, Nagy F, O'Shea MR. Physiological consequences of a peptide cotransmitter in a crayfish nerve-muscle preparation. J Neurosci 1987;7:1769-1779.

39. Anderson J, Bressler BH, Ovalle WK. Functional regeneration in the hindlimb skeletal muscle of the $\mathrm{mdx}$ mouse. J Muscle Res Cell Motil
1988;9:499-515

40. Behm DG, Anderson KG. The role of instability with resistance training. J Strength Cond Res 2006;20:716-722.

41. Galcheva SV, lotova VM, Yotov YT, Grozdeva KP, Stratev VK, Tzaneva VI. Waist circumference percentile curves for Bulgarian children and adolescents aged 6-18 years. Int J Pediatr Obes 2009;4:381-388.

42. Janssen I, Katzmarzyk PT, Boyce WF, Vereecken C, Mulvihill C, Roberts $C$, et al. Comparison of overweight and obesity prevalence in school-aged youth from 34 countries and their relationships with physical activity and dietary patterns. Obes Rev 2005;6:123-132

43. Kelishadi R, Gouya MM, Ardalan G, Hosseini M, Motaghian M, Delavari $A$, et al. First reference curves of waist and hip circumferences in an Asian population of youths: CASPIAN study. J Trop Pediatr 2007:53:158-164.

44. Sung RY, So HK, Choi KC, Nelson EA, Li AM, Yin JA, et al. Waist circumference and waist-to-height ratio of Hong Kong Chinese children. BMC Public Health 2008;8:324.

45. Chen LK, Liu LK, Woo J, Assantachai P, Auyeung TW, Bahyah KS, et al. Sarcopenia in Asia: consensus report of the Asian Working Group for Sarcopenia. J Am Med Dir Assoc 2014;15:95-101. 